Accepted by American Journal of Speech Language Pathology 9/12/2017

\title{
The Effects of Intensive Speech Treatment on Conversational Intelligibility in Spanish Speakers with Parkinson's Disease
}

\author{
Author(s) Gemma Moya-Galé1,3, Alireza Goudarzi², Àngels Bayés ${ }^{3}$, Megan McAuliffe ${ }^{4}$, Bram \\ Bulté $^{5}$, and Erika S. Levy ${ }^{1}$ \\ ${ }^{1}$ Teachers College, Columbia University, New York, New York \\ ${ }^{2}$ RIKEN Brain Science Institute, Saitama, Japan \\ ${ }^{3}$ UParkinson, Centro Médico Teknon, Grupo Hospitalario Quirón, Barcelona \\ ${ }^{4}$ University of Canterbury, Christchurch, New Zealand \\ ${ }^{5}$ Vrije Universiteit Brussel, Belgium
}

Correspondence concerning this article should be addressed to Gemma Moya-Gale, UParkinson, Centro Médico Teknon, Carrer d'Horaci 12, 08022 Barcelona, Spain. E-mail: gm2446@columbia.edu

\begin{abstract}
Purpose: To examine the effects of intensive speech treatment on the conversational intelligibility of Castilian Spanish speakers with Parkinson's disease (PD), as well as on the speakers' self-perceptions of disability.

Method: Fifteen speakers with a medical diagnosis of PD participated in this study. Speech recordings were completed twice before treatment, immediately post-treatment and at a one-month follow-up session. Conversational intelligibility was assessed in two ways - transcription accuracy scores and intelligibility ratings on a 9-point Likert scale. The Voice Handicap Index (VHI) was administered as a measure of self-perceived disability.

Results: Group data revealed that transcription accuracy and median ease-of-understanding ratings increased significantly immediately post-treatment, with gains maintained at the onemonth follow-up. The functional subscale of the VHI decreased significantly post-treatment, suggesting a decrease in perceived communication disability after speech treatment.

Conclusion: These findings support the implementation of intensive voice treatment to improve conversational intelligibility in Spanish speakers with PD with dysarthria as well as to improve the speakers' perception of their daily communicative capabilities. Clinical and theoretical considerations are discussed.
\end{abstract}




\section{Introduction}

Parkinson's disease (PD) affects approximately 150,000 people in Spain (Federación Española de Parkinson, 2010) and four to six million people worldwide. It is the second most common neurodegenerative disease following Alzheimer's disease. In the United States, PD is estimated to affect one million individuals and is rated the $14^{\text {th }}$ leading cause of death (National Parkinson Foundation, 2013). The motor speech disorder of hypokinetic dysarthria is prototypically associated with PD and its effects on various voice parameters (e.g., pitch, loudness, stress) have been reported to negatively impact speakers' intelligibility (Yorkston, Beukelman, Strand, \& Hakel, 2010), which, in turn may compromise this large population's communicative participation (McAuliffe, Baylor, \& Yorkston, 2016).

In a study of the incidence of PD in the US, Hispanics were found to have the highest incidence of the disease, followed by non-Hispanic Whites (Van Den Eeden et al., 2003). Moreover, according to the 2010 U.S. Census Bureau report on the languages spoken in the United States in 2007, of the 55.4 million people who spoke a language other than English at home, 62\% spoke Spanish (U.S. Census Bureau, 2010). Spanish is the second most spoken native language in the world, with over 472 million native speakers. Moreover, Spanish is spoken by almost 567 million people when second language learners are included (Fernández Vítores, 2016). Despite the prevalence of this linguistic group, however, little is known about Spanish dysarthria and even less, about the effects of speech treatment on intelligibility in Spanish.

Intelligibility is a complex construct, influenced by a number of factors, including the complexity of utterances and the environment in which communication takes place. Research studies examining intelligibility in dysarthria secondary to PD commonly undertake intelligibility assessment through estimates of intelligibility and/or transcription tasks focused on words and/or sentences and usually in quiet laboratory conditions. Neel (2009), for example, examined intelligibility in a quiet environment by means of a 7-point rating scale and word transcription accuracy in two separate experiments. Tjaden and Wilding (2004) investigated intelligibility in quiet in speakers with PD and multiple sclerosis (MS) using magnitude estimation of a paragraph excerpt. Sentence transcription and visual analogue scales (usually assessing stimuli in quiet settings) have also been used in the examination of perceived intelligibility (Sussman \& Tjaden, 2012). Although these methods have provided insight into how dysarthric speech in PD is perceived by naïve listeners, they may be limited in their representation of the everyday communication difficulties experienced by those with PD in that conversational speech in noisy environments is more typical of communicative situations than repeated or read utterances in quiet, for example.

One way in which intelligibility can be examined in perhaps more meaningful, real-life contexts (Yorkston, Hakel, Beukelman, \& Fager, 2007) is through the addition of background noise (Fontan, Tardieu, Gaillard, Woisard, \& Ruiz, 2015; Ozimek, Warzybok, \& Kutzner, 2010). Presumably, this approach better simulates communicative deficits experienced by those with dysarthria in everyday listening environments. For example, Tjaden, Sussman, and Wilding (2014) examined scaled intelligibility of utterances produced in three different speaking styles (i.e., clear, loud, and slow) in 16 speakers with PD and 30 speakers with MS. Their sentence productions were then mixed with multitalker babble before presentation to listeners who utilized a continuous visual analogue scale to judge either the speakers' intelligibility or severity. Similarly, Stipancic, Tjaden, and Wilding (2016) examined the orthographic transcription of sentences that were mixed with multitalker babble in a study that compared their objective measures of intelligibility to the subjective measures in Tjaden et al.'s (2014) investigation. 
The current study focused on the outcomes of an intensive speech treatment in a group of Spanish speakers with dysarthria. Conversational speech was chosen for intelligibility analysis because, although less easily controlled experimentally, it represents a more natural communicative condition and is more likely to reflect the true speech characteristics of speakers with PD than more structured speech tasks (Sapir et al., 2007). Because conversational speech is thought to be the most socially-valid communication mode for evaluating intelligibility (Flipsen, 2006) and, thus, may have greater external validity (De Bodt, 2002; Weismer, Jeng, Laures, Kent, \& Kent, 2001), an emotional monologue was selected in this study of treatment-related changes in speakers' intelligibility.

\section{Voice Treatment Speech in PD}

The Lee Silverman Voice Treatment (LSVT LOUD) is an intensive speech treatment that aims to increase vocal intensity in individuals with dysarthria secondary to PD. This treatment has Level I evidence for improving vocal fold function in English speakers with PD (Ramig, Countryman, Thompson, \& Horii, 1995; Ramig et al., 2001). It is based on motor learning principles, mainly specificity, saliency, intensity and repetition, and aims to enhance neural plasticity through the implementation of motor routines (Fox, Ebersbach, Ramig, \& Shapir, 2012; Kleim, Jones, \& Schallert, 2003; Kleim \& Jones, 2008). LSVT LOUD is reported to increase subglottal air pressure and thus increase vocal intensity, improve vocal fold adduction and articulatory movements, and enhance vocal tract configurations in English speakers with PD (Ramig et al., 2001). The resulting physiological changes have been shown to improve vocal quality (Baumgartner, Sapir, \& Ramig, 2001) and articulation (Dromey, Ramig, \& Johnson, 1995), expand fundamental frequency range (i.e., prosodic inflections) and enhance resonance (Ramig et al, 2001).

A few studies report on the effects of LSVT LOUD on speech intelligibility in dysarthria. For example, Ramig et al. (1995) showed significant improvements in pre-to-post intelligibility ratings following LSVT LOUD. In El Sharkawi et al.'s (2002) study of LSVT LOUD effects on swallowing and voice, perceived speech, as measured by speech assessment scales, visual analogue scales and Voice Handicap Index (VHI; Jacobson et al., 1997) scores, trended towards improvement as a function of treatment although the gains were not statistically significant. There has been limited investigation of the outcomes of LSVT LOUD using orthographic transcription tasks, which are considered the gold standard of intelligibility measurement (Fontan et al., 2015; Hustad, 2006; Stipancic et al., 2016). In a single case study, significant increase in words understood (in a sentence task) was observed based on six blinded listeners' ratings pre and post LSVT (Cannito et al., 2008). In a second study of eight participants with PD, Sentence Intelligibility Test (SIT; Yorkston, Beukelman, Hakel, Dorsey, 2007) scores pre- and post-treatment presented in pink noise to 24 listeners increased significantly following treatment (Cannito et al., 2012). To the best of our knowledge, however, only two studies have investigated the effects of intensive speech or voice treatment on conversational intelligibility in English speakers with dysarthria due to PD. In both investigations, preliminary results indicated that the group of 19 speakers who received LSVT LOUD demonstrated significant improvements in conversational intelligibility (Levy, Moya-Galé, Hopf, Forrest, \& Ramig, 2016; Ramig et al., 2015).

Overall, LSVT LOUD results in improvements to voice and phonatory function and preliminary results suggest enhanced intelligibility in English speakers with PD. While the approach is practiced in numerous countries in which English is not the primary language, there has been minimal study of the outcomes of treatment with non-English speakers. Whitehill, Kwan, Lee, and Chow (2011) investigated the effects of LSVT LOUD in 12 Cantonese-speaking individuals with idiopathic PD. Results revealed significant improvements in both vocal loudness and 
intonation. However, lexical tone was already relatively intact at pre-treatment, as measured by tone acoustics (i.e., fundamental frequency configurations) and perceptual analysis (i.e., tone transcription of isolated syllables and identification of incorrect lexical tones in phrases). Intelligibility in connected speech, however, was not measured and disability-based measures were not included in this study. In a study on French dysarthria, Martel Sauvageau, Roy, Langlois, and Macoir (2015) analyzed the effects of LSVT LOUD on vowel articulation and coarticulation in nine speakers of Quebecois French with a medical diagnosis of idiopathic PD. Vowel space and vowel duration increased and greater distinctiveness in consonant-vowel coarticulation patterns was achieved post-treatment. Outcomes of an adaptation of LSVT LOUD have also been examined in speakers of Brazilian-Portuguese. Lemos de Azevedo, Soares de Souza, Marques de Oliveira and Cardoso (2015) examined prosodic parameters in ten Brazilian-Portuguese speakers with PD. Speakers received 16 50- minute sessions twice a week (instead of four 50-minute sessions during four weeks, as described in the original protocol). Results yielded an increase in fundamental frequency measures and intensity and a decrease in measures of duration after the intensive treatment. Again, neither speech intelligibility nor disability-based measures were assessed in these studies.

To the authors' knowledge, the effects of the LSVT LOUD program have not been investigated in Spanish-speaking individuals with PD. Furthermore, overall research on the nature or treatment of Spanish dysarthria secondary to PD is sparse. In his doctoral dissertation, Frass (2003) investigated the relationship between the acoustic variables of voice onset time (VOT), vowel space and second formant (F2) transitions and intelligibility at the single word level in 11 Spanish speakers from Texas and 14 healthy controls, with modest results. In an acoustic study, Gamboa et al. (1997) examined the features of dysarthric speech in 41 Castilian Spanish-speaking individuals with PD who were treated with dopaminergic drugs. Findings indicated increased jitter (i.e., frequency perturbation) and reduced harmonic/noise ratio during sustained vowel phonation, and reduced fundamental frequency range and intensity during sentence production. Similarly, Jiménez- Jiménez et al. (1997) examined voice features in 22 Castilian Spanish-speaking individuals with PD who were not treated with dopaminergic medication (i.e., recorded in their OFF phase). Acoustic analyses also revealed increased jitter and reduced harmonic/noise ratio during sustained vowel phonation, as well as reduced fundamental frequency range in sentence production. Increased shimmer was also reported in these speakers. Despite these studies, however, little is known about speech intelligibility in Spanish speakers with hypokinetic dysarthria and the effects of treatment on their intelligibility.

\section{Aims of the Present Study}

The current study was motivated primarily by the paucity of evidence on which to base speech treatment for Spanish speakers with PD. We examined the changes in conversational intelligibility of native Spanish speakers with PD as a function of LSVT LOUD. This treatment, which emphasizes increased vocal loudness, was selected for two primary reasons: First, LSVT LOUD is the treatment with the strongest evidence (i.e, Ramig et al., 1995; Ramig et al., 2001) to date for improving vocal function in English speakers with dysarthria secondary to PD. Secondly, increased vocal loudness has been shown to improve perceived intelligibility in English speakers with dysarthria (e.g., Levy et al., 2016; Tjaden \& Wilding, 2004). Loud speech has been hypothesized to enhance cues to syllabic stress in English dysarthria (e.g., increasing vocal intensity, improving pitch, changing vowel articulation), potentially facilitating lexical stress segmentation (Lansford, Liss, Caviness, \& Utianski, 2011). If improvements in intelligibility 
occured universally as a function of louder speech, LSVT LOUD would also be expected to improve intelligibility in Spanish and across languages.

Additionally, in the present study the speakers' self-perception of disability (as measured by the VHI) and sound pressure level (SPL) were examined pre- and post-treatment, as well as one-month post-treatment to assess maintenance of any changes. We hypothesized that there would be a significant increase in conversational intelligibility (i.e., percentage of words understood after LSVT LOUD treatment and in intelligibility ratings), as well as in SPL in Spanish dysarthria. We also hypothesized a significant decrease in VHI scores, which would be indicative of an improvement in the speakers' functional communicative abilities, the physical condition of their voice and their emotional state.

More theoretically, examining intelligibility (e.g., as assessed by naïve listeners) in various languages could be of interest to better understand not just the universal deficits of speech production induced by neurological pathologies, but also difficulties induced by the linguistic characteristics and constraints of the particular language studied (Pinto, Chan, Guimarães, RotheNeves, \& Sadat, 2017: Miller \& Lowit, 2014). In addition, examining more global intelligibility changes in Spanish speakers with dysarthria as a function of treatment is a clinically urgent and theoretically relevant first step in such cross-linguistic research.

\section{Method}

This study was approved by the Institutional Review Board at Teachers College, Columbia University and in Barcelona, Spain.

\section{Speakers}

Fifteen adults (10 males and 5 females) with a medical diagnosis of PD and no history of speech or language problems prior to the onset of their disease participated in the study. Speakers' biographical details are presented in Table 1.

Table 1. Biographical details of the 15 speakers with hypokinetic dysarthria

\begin{tabular}{|c|c|c|c|c|c|c|}
\hline Participant & Age & Sex & $\begin{array}{c}\text { Hoehn \& } \\
\text { Yahr stage } \\
\text { (1967) }\end{array}$ & YPD & $\begin{array}{l}\text { Dysarthria } \\
\text { Severity }\end{array}$ & Perceptual Impression \\
\hline P1 & 82 & $\mathrm{~F}$ & 2.5 & 7 & Mild & Hoarseness, reduced volume \\
\hline P3 & 58 & M & 2.0 & 8 & Mild & Hoarseness, monopitch \\
\hline P4 & 59 & M & 2 & 11 & Moderate & $\begin{array}{l}\text { Fast speech, rapid rushes of speech, imprecise } \\
\text { articulation }\end{array}$ \\
\hline P5 & 78 & M & 3 & 8 & Moderate & $\begin{array}{l}\text { Monoloudness, monopitch, reduced volume, } \\
\text { imprecise articulation }\end{array}$ \\
\hline P6 & 69 & $\mathrm{~F}$ & 2.5 & 9 & Mild & Vocal harshness, reduced volume \\
\hline P7 & 80 & M & 2.5 & NA & Moderate & $\begin{array}{l}\text { Monopitch, imprecise articulation, reduced volume, } \\
\text { monoloudness }\end{array}$ \\
\hline P8 & 77 & M & 2 & 14 & Mild-moderate & Reduced volume, short rushes of speech, monopitch \\
\hline P9 & 58 & M & 2 & 15 & Mild & monopitch \\
\hline P10 & 75 & $\mathrm{~F}$ & 2.5 & 6 & Mild & Reduced volume \\
\hline P11 & 69 & M & 2 & 5 & Mild-moderate & Monopitch, monoloudness, reduced volume \\
\hline P12 & 79 & M & 3 & 17 & Moderate & $\begin{array}{l}\text { Fast speech, rapid rushes of speech, imprecise } \\
\text { articulation, reduced volume }\end{array}$ \\
\hline P14 & 64 & M & 2 & 7 & Mild & Hoarseness, instances of pallilalia \\
\hline P15 & 65 & M & 3 & 25 & Moderate & $\begin{array}{l}\text { Breathiness, reduced volume, monoloudness, } \\
\text { monopitch }\end{array}$ \\
\hline P16 & 77 & $\mathrm{~F}$ & 2 & 1 & Mild & Reduced volume \\
\hline P17 & 62 & $\mathrm{~F}$ & 2.5 & 1 & Mild & Reduced volume \\
\hline
\end{tabular}


All were native speakers of Castilian Spanish, ranging in age from 58 to 82 years $(M=$ $70, S D=8$ ). Speakers scored a minimum of $25 / 30$ on the Mini-Mental State Examination (Folstein, Folstein, \& McHugh, 1975; Lobo et al., 1999) in their initial evaluation, suggestive of no cognitive impairment. Their degree of motor severity was categorized as Stages I-III on the Hoehn and Yahr (1967) Scale during their ON phase, as determined by a neurologist. Similarly, speakers scored no higher than 2 (i.e., moderate difficulty with little or no assistance) in the gross motor components (e.g., walking) of Part III of the Unified Parkinson Disease Rating Scale (UPDRS; Goetz et al., 2008) for their motor evaluation and obtained a minimum score of 1 (i.e., mild difficulty) during their ON phase. Presence and severity of hypokinetic dysarthria were determined by two speech-language pathologists, both native speakers of Castilian Spanish, via a consensus rating procedure (McAuliffe, Kerr, Gibson, Anderson, \& LaShell, 2014). Perceptual judgments were based on audio recordings of the speakers' maximum phonation, diadochokinesis, and a brief conversational monologue (Tjaden \& Wilding, 2004).

Hypokinetic dysarthria was operationally defined as the presence of some or all of the following speech characteristics: mono-pitch, mono-loudness, reduced stress and loudness, short rushes of speech, inappropriate silences and/or tremor (Rosen, Kent, Delaney, \& Duffy, 2006). Speakers' severity of dysarthria was categorized as mild $(n=8)$, mild to moderate $(n=2)$ and moderate $(n=5)$. All speakers were neuropharmacologically stable during treatment. Those who had undergone surgical procedures such as deep brain stimulation (DBS) were excluded from this study.

Speakers were recruited from UParkinson, a specialized unit for PD research and treatment, at the Teknon Medical Center in Barcelona, Spain. They received intensive speech treatment from the first author and all services were free of charge. Recruitment took place in Spain because it provided a good opportunity to establish dialectical homogeneity among the speakers with PD.

\section{Procedure}

Speech testing took place at the Laboratory of Phonetics of the University of Barcelona on four occasions - one month and one week before initiation of treatment, one week post-treatment, and one month after the post-treatment test. At their first assessment, speakers completed a language background questionnaire at the beginning of the recording session. At each session they underwent recordings of their conversational speech and completed the Spanish-validated version of the Voice Handicap Index (VHI; Núñez-Batalla et al., 2007). Full details are provided below.

For the conversational speech recording, speakers wore an EMW Omnidirectional Lavalier microphone taped to their forehead and secured with a headband. A mouth-to-microphone distance of $8 \mathrm{~cm}$ was maintained constant across speakers. The microphone signal passed through a LBS Whirlwind Splitter and a Focusrite Scarlett $2 \mathrm{i} 2$ audio interface and was recorded into a digital (ZOOM H4n handy) recorder at a sampling rate of $48 \mathrm{kHz}$ with 16 bits of quantization. The input level was not changed throughout the entire study. A calibration tone was generated at the beginning and at the end of each recording session with a KORG LCA-120 Chromatic Tuner for calculation of SPL and noted on a Galaxy CM140 sound level meter (SLM). For each calibration tone, the forehead microphone and the SLM were positioned $8 \mathrm{~cm}$ from the mouth of a Styrofoam head, modeling the actual recording conditions for each speaker (Fox \& Boliek, 2012).

During the testing sessions, speakers were recorded producing an emotional monologue 
in which they described a happy day in their lives for approximately 60 seconds (e.g., Ramig et $a l .$, 1995). The instructions were: 'Ahora quiero que piense en un día o momento feliz en su vida y que lo comparta conmigo' [Now I want you to think of a happy day or moment in your life and share it with me]. The principal investigator, who delivered speech treatment, did not collect post-treatment data in order to avoid speakers' familiarity with the clinician (Kapsner-Smith et al., 2014).

The VHI was administered to provide information on the impact of the speakers' communication disorder on their quality of life and activities of daily living. This questionnaire was selected because of its ecological validity as a measure of self-report (Kapsner-Smith, Hunter, Kirkham, Cox, \& Titze, 2014).

\section{Treatment Protocol}

Participants engaged in treatment in their ON phase because motor learning has been found to depend not only on task-training per se (i.e., speech treatment exercises) but also on dopamine effects (Beeler et al., 2010; Kang \& Auinger, 2012). The speech intervention for this study, LSVT LOUD, followed the protocol as described in Ramig et al. (1995) and was implemented in four individual and consecutive 60-minute sessions a week for four weeks (i.e., total number of sessions $=16$ ). Following the treatment protocol, maximum performance tasks (i.e., maximum sustained phonation of 'ah' and maximum fundamental frequency range), and reading of functional phrases constituted the first half of each session. Language tasks, which varied daily, constituted the second half of the session and were constructed following a weekly hierarchical order of language complexity (i.e., single words/phrases, sentences, paragraph and conversational level). Treatment materials were tailored toward each speaker's personal interests in order to increase treatment saliency.

Augmented feedback, a component of the LSVT LOUD protocol, was provided to participants through various instruments (e.g., voice recorder, stop watch, voice mail) in order to enhance speakers' performance. As sensory dysfunction is a common characteristic in PD (Nolano et al., 2008), knowledge of results was provided as a substitute for the missing task-intrinsic feedback that is required to learn the target skill (Magill, 2011). All speakers received daily homework and carry-over tasks. Treatment sessions were video-recorded on a Canon VIXIA HF R500 Digital Camcorder and reviewed by a second speech-language pathologist in order to ensure treatment fidelity (Moncher \& Prinz, 1991), strengthening the study's internal validity.

\section{Listeners}

A total of 21 listeners (nine men and 12 women) were recruited to complete the two intelligibility tasks. The listeners' average age was 38.1 years $(S D=14.2$, range $=18-57$ years $)$. Listeners were native speakers of Castilian Spanish and reported no history of speech, language or hearing disorders. Additionally, none of them reported having had experience with motor speech disorders. They all passed a bilateral pure-tone hearing screening at 25dB at 500, 1000, 2000 and $4000 \mathrm{~Hz}$ (American National Standard Institute, 2010) and completed a language background questionnaire prior to their participation in the study.

The intelligibility investigation was based on samples from the emotional monologue produced by the speakers with PD. Listeners were seated in front of a MacBook Pro laptop with Mac OSX 10.10.3 and wore Sennheiser HD 280 pro headphones at a comfortable listening level. Two tasks were conducted to examine speech intelligibility: transcription accuracy and intelligibility ratings, as described below.

\section{Stimulus preparation}


The stimuli $(n=270)$ consisted of grammatically correct six- to nine-word utterances (Beijer, Clapham, \& Rietveld, 2012). Utterances were selected approximately $20 \mathrm{sec}$ into the speech sample in order to eliminate potential effects that tend to be associated with the beginning and end of the speech signal (Turner, Tjaden, \& Weismer, 1995). Three utterances per data collection point for each speaker (i.e., two baselines, one immediate post-test, and one-month follow-up) were included in these tasks, totaling 12 utterances per speaker. Speech samples were discarded if they contained extraneous noise (Levy, Chang, Ancelle, \& McAuliffe, 2017), most often due to dyskinesias. Although some degree of experimental control was relinquished when using utterances from a spontaneous speech sample, semantically unpredictable utterances of the length selected for the present study have been found to be suitable intelligibility measures for efficacy research (Beijer et al., 2012). For stimulus verification before the selected utterances were embedded in noise, two trained scorers transcribed them, attaining $100 \%$ accuracy. Utterances were randomized across time point and speakers and presented in five blocks of 54 items. Differences in speaking rate between the samples were not eliminated.

Utterances were then embedded in six-talker babble (Simpson \& Cooke, 2005; Van Engen \& Bradlow, 2007) using Matlab software. This type of noise was chosen to increase ecological validity (Cullington \& Zeng, 2008; Fontan et al., 2015; Wilson, Abrams, \& Pillion, 2003) because communicative environments that include background talkers are frequently experienced by listeners. The multi-talker babble consisted of six native Spanish speakers (three males and three females) speaking about different topics in order to approach recreation of listeners' daily communication environment (to the extent possible in this experimental setting). The recording for each babble talker was processed separately so that the noise component had the intensity of $-25 \mathrm{~dB}$. Possible pauses between words in the babble were also deleted. The individual recordings were subsequently mixed together to create a single six-talker noise. In order to ensure that the noise intensity was constant at all times, the noise was divided in consecutive windows of 0.025 seconds, each of which was adjusted to have the intensity of $-25 \mathrm{~dB}$. The resulting noise file was then adjusted to have the same intensity as each pre-treatment utterance to achieve $0 \mathrm{~dB}$ SNR for that utterance. Finally, the noise levels for the post-test and follow-up samples were maintained constant relative to the pre-test noise levels of each utterance in order to examine treatment effects in the same noise environment. Multitalker babble has been studied in the literature at various SNRs (Simpson \& Cooke, 2005; Van Engen \& Bradlow, 2007). Noise levels for these speakers were piloted at $-5 \mathrm{~dB},-2 \mathrm{~dB}, 0 \mathrm{~dB},+2 \mathrm{~dB}$ and $+5 \mathrm{~dB}$ SNR, and the resulting ceiling and floor effects on the intelligibility measures were examined. A final SNR of $0 \mathrm{~dB}$ was selected to best capture the perceived range of severity of the speakers' dysarthria.

\section{Listener Tasks: Transcription Accuracy and Intelligibility Ratings}

Listeners were instructed to type orthographic (i.e., word-by-word) transcriptions of the recordings on an Excel spreadsheet. They were further instructed to provide an estimate of intelligibility for the target utterances using a 9-point Likert scale (i.e., $1=$ nada inteligible [not at all intelligible] and $9=$ completamente inteligible [completely intelligible]). The following instructions were provided verbally and also appeared on the computer screen: 'Por favor transcriba esta muestra de voz. Las transcripciones son ortográficas (con letras normales). En una escala del 1 al 9, valore cómo de inteligible es la frase que acaba de transcribir' [Please transcribe this speech sample. Transcriptions are orthographic (with normal letters). On a scale from 1-9, rate the intelligibility of this sentence you just transcribed]. A practice phase was included for task familiarization purposes. In this phase, listeners were presented with six utterances (of speakers with PD and stimuli not included in the experiment) and completed the transcription and rating 
tasks as detailed above. They were given the opportunity to ask clarification questions to the investigator during familiarization. The practice and experimental tasks were self-paced and took approximately 90 minutes to complete. Listeners were given a 2-min break after each block of stimuli if needed. Listeners heard each utterance only once to avoid learning effects (Wilson, Bell, \& Koslowski, 2003).

Six utterances were replayed to each listener (with intervals of 54 stimuli) to assess intra-listener reliability. Reliability was high for all listeners, with Cohen's linearly weighted kappa ranging between 0.83 and 1 for ratings and between 0.875 and 1 for transcription accuracy. Although there was some variation in the transcription ability of the listeners, there were no clear positive or negative outliers. The average transcription accuracy per listener ranged from $42.05 \%$ to $58.36 \%$, with a mean of $49.67 \%(S D=4.38)$. Average ratings ranged from 4.18 to $6.44(M=$ 5.05; $S D=0.49$ ).

\section{Statistical Analysis}

A series of general linear mixed-effects models was fitted to the data set to test for significant differences in transcription accuracy, intelligibility ratings, SPL and VHI scores across data collection points. The trichotomous categorical variable 'dysarthria severity' was included in the model as predictor (with categories 'mild', 'mild to moderate' and 'moderate'). The first pretreatment session (for time) and 'moderate' (for severity) were coded as reference categories. Random effects were restricted to random intercepts (for each speaker). Approximate F-tests were used to verify whether the estimated fixed effects were statistically significant.

The percentage of correctly transcribed words and listeners' ratings of each utterance were averaged per speaker and data collection point from a total of 3780 observations (15 speakers $\mathrm{x} 4$ data points $\mathrm{x} 3$ utterances $\mathrm{x} 21$ listeners). First an average of the 21 listener ratings was calculated per sentence, and then the average of the three sentences per data collection point was determined for each speaker. The pairwise inter-listener reliability was high for both the percentage of correctly understood words $(n=60 ; 0.667<r<0.906 ; p<0.01)$ and the ratings $(n=60$; $0.702<r<0.894 ; p<0.01)$. Likewise, SPL was averaged over the three sentences per speaker and data collection point.

\section{Results}

The findings on transcription accuracy, intelligibility ratings, and SPL are described below, followed by results of the VHI.

\section{Transcription, Ratings, and Sound Pressure Level}

Table 2 shows the mean percentage (and standard deviation) of correctly transcribed words, intelligibility ratings and SPL per data collection point. Detailed parameter estimates and fit statistics of the mixed effects models are provided in Table 3 , and the results of the approximate F-tests of the fixed effects are given in Table 4. 
Table 2. Means, standard deviations and range for transcription accuracy scores, intelligibility ratings, and sound pressure level

\begin{tabular}{|c|c|c|c|c|c|c|}
\hline \multirow[b]{2}{*}{ Time } & \multicolumn{2}{|c|}{$\begin{array}{c}\text { Transcription } \\
\text { Accuracy } \\
(\%)\end{array}$} & \multicolumn{2}{|c|}{$\begin{array}{c}\text { Intelligibility } \\
\text { Ratings } \\
(1-9)\end{array}$} & \multicolumn{2}{|c|}{$\begin{array}{c}\text { Sound } \\
\text { Pressure Level } \\
\text { (dB) }\end{array}$} \\
\hline & $\begin{array}{c}M \\
(S D)\end{array}$ & $\begin{array}{l}\min \\
\max \end{array}$ & $\begin{array}{c}M \\
(S D)\end{array}$ & $\begin{array}{l}\min \\
\max \end{array}$ & $\begin{array}{c}M \\
(S D)\end{array}$ & $\begin{array}{l}\min \\
\max \end{array}$ \\
\hline Pretest 1 & $\begin{array}{c}32.28 \\
(31.81)\end{array}$ & $\begin{array}{c}0.18 \\
89.64\end{array}$ & $\begin{array}{c}3.61 \\
(2.35)\end{array}$ & $\begin{array}{l}1.17 \\
7.83\end{array}$ & $\begin{array}{l}65.03 \\
(5.11)\end{array}$ & $\begin{array}{l}57.19 \\
74.40\end{array}$ \\
\hline Pretest 2 & $\begin{array}{c}28.55 \\
(27.17)\end{array}$ & $\begin{array}{c}0.40 \\
83.02\end{array}$ & $\begin{array}{c}3.51 \\
(1.98)\end{array}$ & $\begin{array}{l}1.21 \\
7.17\end{array}$ & $\begin{array}{l}64.72 \\
(3.76)\end{array}$ & $\begin{array}{l}58.00 \\
70.20\end{array}$ \\
\hline $\begin{array}{l}\text { Immediate } \\
\text { post }\end{array}$ & $\begin{array}{c}71.73 \\
(26.68)\end{array}$ & $\begin{array}{c}16.47 \\
100\end{array}$ & $\begin{array}{c}6.75 \\
(1.92)\end{array}$ & $\begin{array}{l}2.84 \\
8.78\end{array}$ & $\begin{array}{l}69.15 \\
(3.53)\end{array}$ & $\begin{array}{l}61.40 \\
75.60\end{array}$ \\
\hline Follow-up & $\begin{array}{c}66.08 \\
(28.22)\end{array}$ & $\begin{array}{c}2.34 \\
97.53\end{array}$ & $\begin{array}{c}6.33 \\
(2.03)\end{array}$ & $\begin{array}{l}1.86 \\
8.43\end{array}$ & $\begin{array}{l}68.27 \\
(3.73)\end{array}$ & $\begin{array}{l}60.70 \\
75.20\end{array}$ \\
\hline
\end{tabular}

Transcription accuracy increased significantly as a function of treatment $(p<0.0001)$. The average transcription accuracy scores for speakers with PD were $32.28 \%(S D=31.81 \%$; range: $0.18 \%-89.64 \%)$ for the first pre-treatment and $28.55 \%(S D=27.17 \%$; range: $0.40 \%-$ $83.02 \%$ ) for the second pre-treatment sessions. Performance did not differ significantly at baselines $(p>0.05)$, as indicated by approximate F-tests (see Table 4). The average transcription scores immediately post-treatment were $71.73 \%(S D=26.68 \%$; range: $16.47 \%-100 \%)$ and $66.08 \%(S D=28.22 \%$; range: $2.34 \%-97.53 \%)$ at the one-month follow-up, with no significant difference in performance between the two post-treatment sessions. $(p>0.05)$. Intelligibility ratings also increased significantly after treatment $(p<0.0001)$. The utterances by the speakers with PD were rated $3.61(S D=2.35$; range: $1.17-7.83)$ on average at the first pre-treatment and 3.51 $(S D=1.98$; range: $1.21-7.17)$ at the second pre-treatment session. There was no significant difference in performance between both baseline pre-treatment sessions $(p>0.05)$. The average intelligibility ratings after the treatment were $6.75(S D=1.92$; range: $2.84-8.78)$ for the immediate post-test and $6.33(S D=2.03$; range: $1.86-8.43)$ for the one-month follow-up. No significant difference was found between the ratings immediately post-treatment and the ratings one month afterwards $(p>0.05)$. Sound pressure levels followed a similar pattern to both measures of intelligibility. The mean SPL increased significantly as a function of treatment $(p<0.001)$. SPL at the first pretest was $65.03 \mathrm{~dB}(S D=5.11$; range: $57.19-74.40)$ on average and $64.72 \mathrm{~dB}$ $(S D=3.76$; range: $58.00-70.20)$ at the second pretest. The difference in SPL between the two pretests was not significant $(p>0.05)$. Immediately post-treatment, the average SPL increased to $69.15 \mathrm{~dB}(S D=3.53$; range: $61.40-75.60)$, and one month later SPL was $68.27 \mathrm{~dB}(S D=3.73$; range: $60.70-75.20)$. No significant difference in SPL was found between the immediate and the delayed post-test $(p>0.05)$.

The estimates for the categorical variable of dysarthria severity are presented in Table 3 below. Transcription accuracy scores for speakers with mild dysarthria were estimated to be 
39.18 percentage points higher than for those diagnosed with moderate dysarthria, and 11.50 percentage points higher compared to speakers with mild to moderate dysarthria. As for intelligibility ratings, speakers with mild dysarthria received, on average, a rating 3.14 points higher than the rating obtained by individuals with moderate dysarthria, and 1.04 points higher than those with mild to moderate dysarthria. Furthermore, average SPL for moderate dysarthria was 5.13 $\mathrm{dB}$ lower than the SPL of mild dysarthria and $2.57 \mathrm{~dB}$ lower than that of mild to moderate dysarthria.

Table 3. Parameter estimates and fit statistics mixed effects models for transcription accuracy, intelligibility ratings and sound pressure level

\begin{tabular}{|c|c|c|c|c|c|c|}
\hline & \multicolumn{2}{|c|}{$\begin{array}{l}\text { Transcription } \\
\text { Accuracy (\%) }\end{array}$} & \multicolumn{2}{|c|}{$\begin{array}{c}\text { Intelligibility } \\
\text { Ratings }\end{array}$} & \multicolumn{2}{|c|}{$\begin{array}{c}\text { Sound } \\
\text { Pressure Level }\end{array}$} \\
\hline & Estimate & SE & Estimate & SE & Estimate & SE \\
\hline \multicolumn{7}{|l|}{ Fixed effects } \\
\hline Intercept & 7.69 & 8.51 & $1.65^{* *}$ & 0.57 & $61.95^{* *}$ & 1.30 \\
\hline \multicolumn{7}{|l|}{ Time } \\
\hline Pretest 1 & 0 & . & 0 & . & 0 & . \\
\hline Pretest 2 & -3.73 & 6.42 & -0.09 & 0.43 & -0.31 & 0.94 \\
\hline Immediate post & $39.44^{* *}$ & 6.42 & $3.15^{* *}$ & 0.43 & $4.12^{* *}$ & 0.94 \\
\hline Follow-up & $33.80^{* *}$ & 6.42 & $2.72^{* *}$ & 0.43 & $3.25^{* *}$ & 0.94 \\
\hline \multicolumn{7}{|l|}{ Dysarthria severity } \\
\hline Mild & $39.18^{* *}$ & 9.62 & $3.14^{* *}$ & 0.65 & $5.13^{* *}$ & 1.49 \\
\hline Mild to moderate & 27.68 & 14.12 & $2.10^{*}$ & 0.95 & 2.57 & 2.18 \\
\hline Moderate & 0 & . & 0 & . & 0 & . \\
\hline \multicolumn{7}{|l|}{ Variance components } \\
\hline Speaker & $207.48^{*}$ & 117.42 & $0.94^{*}$ & 0.53 & $5.14^{*}$ & 2.80 \\
\hline Residual & $308.64^{* *}$ & 67.35 & $1.39^{* *}$ & 0.30 & $6.66^{* *}$ & 1.45 \\
\hline REML log-likelihood & \multicolumn{2}{|c|}{493.7} & \multicolumn{2}{|c|}{202.0} & \multicolumn{2}{|c|}{287.8} \\
\hline${ }^{*} p<0.05 ;{ }^{* *} p<0.01$ & & & & & & \\
\hline
\end{tabular}


Table 4. Approximate F-tests of fixed effects

$$
F_{(1,42)} \quad \operatorname{Pr}>F
$$

Transcription Accuracy

\begin{tabular}{lcc}
\hline Pre1 vs Pre2 & 0.34 & 0.564 \\
Pre1 vs Immediate post & 37.80 & $<0.0001$ \\
Pre1 vs Follow-up & 27.76 & $<0.0001$ \\
Pre2 vs Immediate post & 45.29 & $<0.0001$ \\
Pre2 vs Follow-up & 34.23 & $<0.0001$ \\
Immediate vs Follow-up & 0.77 & 0.384 \\
Ratings & & \\
\hline Pre1 vs Pre2 & .05 & 0.830 \\
Pre1 vs Immediate post & 53.50 & $<0.0001$ \\
Pre1 vs Month follow-up & 32.92 & $<0.0001$ \\
Pre2 vs Immediate post & 56.71 & $<0.0001$ \\
Pre2 vs Follow-up & 42.70 & $<0.0001$ \\
Immediate vs Follow-up & 0.99 & 0.325 \\
& & \\
\end{tabular}

Sound pressure level

\begin{tabular}{lcc} 
Pre1 vs Pre2 & 0.11 & 0.746 \\
Pre1 vs Immediate post & 19.12 & $<0.0001$ \\
Pre1 vs Follow-up & 11.87 & 0.001 \\
Pre2 vs Immediate post & 22.08 & $<0.0001$ \\
Pre2 vs Follow-up & 14.22 & 0.0005 \\
Immediate vs Follow-up & 0.86 & 0.359 \\
\hline
\end{tabular}




\section{Voice Handicap Index}

VHI scores per subscale (i.e. functional, physical and emotional) were calculated following standard procedures. Missing responses were imputed using the mean score obtained for the other questions within the relevant category. Table 5 shows the mean scores and standard deviations (SD) for the three subscales of the VHI as well as the total score for the three data collection points. Note that the VHI was completed at only three different occasions (i.e., not at the time of the second pretest). The parameter estimates for the mixed effects model are provided in Table 6.

Table 5. Mean and total scores (with standard deviation) for the three VHI subscales

\begin{tabular}{lcccccccc}
\hline & \multicolumn{2}{c}{ Functional } & \multicolumn{2}{c}{ Physical } & \multicolumn{2}{c}{ Emotional } & \multicolumn{2}{c}{ Total score } \\
\hline Time & $\boldsymbol{M}$ & $\boldsymbol{S D}$ & $\boldsymbol{M}$ & $\boldsymbol{S D}$ & $\boldsymbol{M}$ & $\boldsymbol{S D}$ & $\boldsymbol{M}$ & $\boldsymbol{S D}$ \\
Pre & 15.14 & 10.73 & 13.87 & 8.69 & 8.53 & 10.75 & 37.54 & 27.67 \\
ImmPost & 10.55 & 8.53 & 11.90 & 9.71 & 7.08 & 8.74 & 29.53 & 24.37 \\
Month & 11.49 & 9.06 & 11.61 & 7.39 & 6.88 & 8.08 & 29.98 & 22.27 \\
\hline
\end{tabular}

Mean scores post-treatment were lower than at pre-test in all three subscales. Large standard deviations are indicative of high inter-individual variation. For the functional subscale, post-treatment values showed a significant decrease compared to pre-treatment values $(p<0.05)$, indicating an improvement in self-perceived communicative abilities as a function of treatment. The mean score at pre-test was $15.14(S D=10.73)$, compared to $10.55(S D=8.53)$ immediately post-treatment and $11.49(S D=9.06)$ at follow-up. The difference between the two post-treatment scores was not significant $(p>0.05)$. On the physical subscale, the mean score at pre-treatment was $13.87(S D=8.69)$; immediately post-treatment, $11.90(S D=9.71)$ and $11.61(S D=$ $7.39)$ at follow-up. None of these differences was significant $(p>0.05)$. Changes pre-to-posttreatment in the emotional subscale were not significant either $(p>0.05)$, with the mean score at pre-treatment being $8.53(S D=10.75)$; immediately post-treatment, $7.08(S D=8.74)$ and 6.88 $(S D=8.08)$ at follow-up. The difference pre-to-post-treatment for the total VHI score was also not significant $(p>0.05)$.

The parameter estimates for dysarthria severity indicated that speakers with moderate dysarthria scored 15.82 points higher on average than those with mild dysarthria in the functional subscale, and 5.44 points higher than individuals with mild to moderate dysarthria. Scores in the physical subscale were estimated to differ by 12.79 points between speakers with moderate and mild dysarthria, and only by 3.50 between those with a moderate and mild to moderate speech disorder. On the emotional subscale these differences were 9.66 and 0.26 points, respectively. In total, the average score of speakers with moderate dysarthria was estimated to be 38.26 points 
higher than for speakers with mild dysarthria, and 8.69 points higher compared to individuals with mild to moderate dysarthria.

Table 6. Parameter estimates for the mixed effects model in the Voice Handicap Index

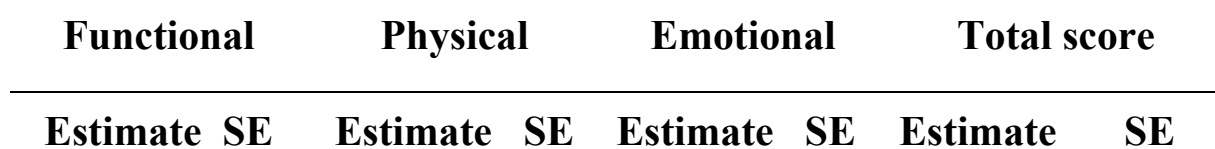

\begin{tabular}{|c|c|c|c|c|c|c|c|c|}
\hline \multicolumn{9}{|l|}{ Fixed effects } \\
\hline Intercept & $24.30^{* *}$ & 2.30 & $21.15^{* *}$ & 2.39 & $13.65^{* *}$ & 3.06 & $59.11^{* *}$ & 6.67 \\
\hline \multicolumn{9}{|l|}{ Time } \\
\hline Pre & 0 & . & 0 & . & 0 & . & 0 & . \\
\hline Immediate post & $-4.59^{*}$ & 1.67 & -1.97 & 1.85 & -1.45 & 2.38 & -8.01 & 4.70 \\
\hline Follow-up & $-3.65^{*}$ & 1.67 & -2.26 & 1.85 & -1.65 & 2.38 & -7.56 & 4.70 \\
\hline \multicolumn{9}{|l|}{ Dysarthria severity } \\
\hline Mild & $15.82^{* *}$ & 2.66 & $12.79^{* *}$ & 2.72 & $-9.66^{* *}$ & 3.49 & $-38.26^{* *}$ & 7.77 \\
\hline Mild to moderate & -5.44 & 3.90 & -3.50 & 3.99 & 0.26 & 5.12 & -8.69 & 11.40 \\
\hline Moderate & 0 & . & 0 & . & 0 & . & 0 & . \\
\hline \multicolumn{9}{|l|}{ Variance components } \\
\hline Speaker & 14.77 & 9.08 & 14.25 & 9.57 & 23.30 & 15.76 & $130.32^{*}$ & 77.17 \\
\hline Residual & $21.01^{* *}$ & 5.62 & $25.55^{* *}$ & 6.83 & $42.54^{* *}$ & 11.37 & $165.64^{* *}$ & 44.27 \\
\hline REML log-likelihood & \multicolumn{2}{|c|}{260.9} & \multicolumn{2}{|c|}{266.9} & \multicolumn{2}{|c|}{287.2} & \multicolumn{2}{|c|}{344.4} \\
\hline${ }^{*} p<0.05 ;{ }^{* *} p<0.01$ & & & & & & & & \\
\hline
\end{tabular}

\section{Discussion}


To the best of our knowledge, this study is the first to examine the effects of intensive voice treatment on conversational intelligibility in Spanish speakers with dysarthria secondary to PD. Overall, significant increases in intelligibility were observed following treatment, as measured by listeners' transcription accuracy and median intelligibility ratings. In tandem, significant decreases in VHI scores were observed - evidence of improvements in functional communication following treatment.

\section{Overall Intelligibility}

LSVT LOUD resulted in significant improvements (from baseline) in intelligibility, as measured by blinded listeners' orthographic transcriptions and ratings of intelligibility at two different time points - immediately post-treatment and at a one-month follow-up. When a 5\% change in sentence intelligibility is obtained in settings with adverse listening conditions, it is likely considered to be clinically meaningful (Stipancic et al., 2016; Tjaden, Kain, \& Lam, 2014). Consequently the intelligibility gain of more than 30 percentage points in transcription accuracy and of three points in median ratings pre-to-post treatment in the present study's sentences in noise represents a substantial gain in intelligibility for our speakers. Moreover, because transcription accuracy is considered the gold standard measure for assessing intelligibility (Fontan et al., 2015; Hustad, 2006; Stipancic et al., 2016), the increase in accuracy scores post-treatment is of clinical relevance. Thus, the treatment shows promise for increasing communicative skills in Castilian Spanish speakers.

Although studies on LSVT LOUD have been conducted in a few other languages (e.g., Hsu, 2017; Lemos et al., 2015; Martel Sauvageau et al., 2015), these have primarily focused on the analysis of acoustic parameters pre- and post-treatment. The increase in conversational intelligibility both in English and in Spanish dysarthria secondary to PD following LSVT LOUD treatment could reflect different, potentially language-universal factors (Pinto et al., 2017). First, improved audibility yielded by increased vocal loudness is hypothesized to enhance cues to syllabic stress, thus potentially facilitating lexical segmentation of the degraded speech signal (Lansford et al., 2011). Second, Cannito et al. (2012) hypothesized that this treatment may also induce changes in the spectral features at the voice source, and that such changes could impact intelligibility. In other words, alterations in formant relationships pre- to post-treatment could result in increased amplitude of the harmonic frequencies beyond the F0 or first harmonic (Cannito, Buder, \& Chorna, 2005). These spectral changes would then lead to more intense formant peaks and to narrower formant bandwidths (Cannito et al., 2012), the latter being associated with increased vowel identification (Hawks, Fourakis, Skinner, \& Holden, 1997). Consequently, all the aforementioned changes at the voice source would result in increased saliency of acoustic cues (Cannito et al., 2008) that would contribute to increased speech intelligibility.

The present study provides preliminary support for this treatment technique for Spanish dysarthria at a complex linguistic level, i.e., conversation, which, to date, has been under-researched in any language. If the present findings are supported by future studies of speakers of other languages (and other Spanish dialects), this could indicate that LSVT LOUD may be beneficial to communication cross-linguistically.

\section{Voice Handicap Index}

Results from the VHI following LSVT LOUD revealed a reduction in mean scores for all three subscales: functional, physical, and emotional, suggesting a decrease in perceived disability. Pre- to post-treatment changes were significant only for the functional subscale, indicating an improvement in the speakers' daily communication abilities, such as being understood in noisy 
environments or interacting more with their family and friends. Pre-treatment scores for the physical and emotional subscales, however, were already low (i.e., not perceived as very disabled) for most speakers in this group, as their primary complaint before initiation of speech treatment was the impact of their reduced vocal volume in their daily lives, rather than on their physical or emotional wellbeing. These findings cannot be compared to the seminal LSVT study (Ramig et al., 2001), as those participants did not complete the VHI. Perceptions of voice handicap were analyzed in Spielman, Ramig, Mahler, Halpern, and Gavin's study (2007), in which they explored an extended treatment version of LSVT LOUD (LSVT-X) in 12 speakers with PD. In this treatment protocol, intensive therapy sessions to improve vocal function were provided twice a week for eight weeks. Their group data findings showed an improvement in VHI scores, which was significant for four of the speakers.

\section{Limitations and Directions for Future Research}

The present study provides preliminary support for the implementation of LSVT LOUD for increasing conversational intelligibility in Castilian Spanish speakers with dysarthria. However, future studies need to examine the effects of speech treatment with a larger sample of participants and a more homogeneous number of severity subgroups. It is also suggested that future studies with the same population include different SNRs to further explore intelligibility in noise in Spanish. It is hypothesized that similar results may be obtained in other Spanish dialects, such as those spoken in the United States (e.g., Puerto Rican or Mexican) because treatment-related changes are rooted in the voice source (Cannito et al., 2012). Future research, however, should also be conducted in other Spanish dialects in order to verify whether our current findings can be generalized to other varieties of this language.

This investigation did not include speakers with severe dysarthria; thus, it remains to be determined whether current findings would also apply to speakers with more severe dysarthria and whether gains, if any, would be maintained past the completion of treatment. Positive effects of LSVT LOUD were found with hypokinetic dysarthria secondary to PD; but other types of dysarthria in Spanish speakers should also be considered in future studies. Finally, a limitation of the use of (the presumably more ecologically valid) conversational speech is that some stimulus control was relinquished as utterances varied linguistically within and across speakers.

In conclusion, this study found that intensive voice treatment improved intelligibility at the conversational level in the current group of Spanish speakers with hypokinetic dysarthria secondary to PD. LSVT LOUD also benefitted speakers' perception of their daily communicative capabilities. These results contribute to a better understanding of the effects of LSVT LOUD on intelligibility and communication disability in speakers of Spanish with PD. Additionally, these data may help speech-language pathologists reach informed clinical decisions when treating Spanish-speaking patients with this motor speech disorder.

\section{Acknowledgements}

We sincerely thank the participants with Parkinson's disease, their families, UParkinson, the team at the Laboratory of Phonetics at the University of Barcelona and the listeners who took part in this project. We also extend our heartfelt thanks to Drs. Lisa Edmonds, Elizabeth Tipton and Karen Froud and to Valerie Levy for their insightful feedback. 


\section{References}

ANSI. (2010). Specification for Audiometers. ANSI Report No. S3.6-2010, New York, NY: ANSI.

Baumgartner, C. A., Sapir, S., \& Ramig, L. O. (2001). Voice quality changes following phonatory-respiratory effort treatment (LSVT) versus respiratory effort treatment for individuals with Parkinson Disease. Journal of Voice, 15, 105-114.

Beeler, J. A., Huang Cao, Z. F., Kheirbek, M. A., Ding, Y., Koranda, J., Murakami, M., Kang, U. J., \& Zhuang X. (2010). Dopamine-dependent motor learning: Insight into levodopa's long-duration response. Annals of Neurology, 67, 639-647.

Beijer, L. J., Clapham, R. P., \& Rietveld, A. C. M. (2012). Evaluating the suitability of orthographic transcription and intelligibility scale rating of semantically unpredictable sentences (SUS) for speech training efficacy research in dysarthric speakers with Parkinson's Disease. Journal of Medical Speech-Language Pathology, 20, 17-34.

Cannito, M. P., Buder, E. H., Chorna, L. B. (2005). Spectral amplitude measures of adductor spasmodic dysphonic speech. Journal of Voice, 19, 391-410.

Cannito, M. P., Suiter, D. M, Chorna, L., Beverly, D., Wolf, T., \& Watkins, J. (2008). Speech intelligibility in a speaker with ideopathic Parkinson's Disease before and after treatment. Journal of Medical Speech-Language Pathology, 16, 207-212.

Cannito, M. P., Suiter, D. M., Beverly, D., Chorna, L., Wolf, T, \& Pfeiffer, R. M. (2012). Sentence intelligibility before and after voice treatment in spealers with idiopathic Parkinson's disease. Journal of Voice, 26, 214-219.

Cullington, F. E., \& Zeng, F.-G. (2008). Speech recognition with varying numbers and types of competing talkers by normal- hearing, cochlear-implant, and implant simulation subjects. The Journal of the Acoustical Society of America, 123, 450-461.

De Bodt, M. S., Hernandez-Diaz Huici, M. E., \& Van de Heyning, P. H. (2002). Intelligibility as a linear combination of dimensions in dysarthric speech. Journal of Communication Disorders, 35, 283-292.

Dromey, C., Ramig, L. O., \& Johnson, A. B. (1995). Phonatory and articulatory changes associated with increased vocal intensity in Parkinson Disease: A case study. Journal of Speech and Hearing Research, 38, 751-764.

Duffy, J. R. (2013). Motor speech disorders: Substrates, differential diagnosis, and management. St. Louis, MO: Mosby.

El Sharkawi, A., Ramig, L., Logemann, J. A., Pauloski, B. R., Rademaker, A. W., Smith, C. H., Pawlas, A., Baum, S., \& Werner, C. (2002). Swallowing and voice effects of Lee Silverman Voice Treatment (LSVT LOUD): A pilot study. Journal of Neurology, Neurosurgery, and Psychiatry, 72, 31-36.

Federación Española de Párkinson. (2010). Memoria de Actividades. http://www.fedesparkinson.org/upload/20110316035435.pdf

Fernández Vítores, D. (2016). El español: Una lengua viva. Informe 2016. Madrid: Instituto Cervantes.

Flipsen, P. (2006). Measuring the intelligibility of conversational speech in children. Clinical Linguistics \& Phonetics, 20, 303-312.

Folstein, M. F., Folstein, S. E., \& McHugh, P. R. (1975). Mini-mental state: A practical method for grading the cognitive state of patients for the clinician. Journal of Psychiatric Research, 12, 189-198. 
Fontan, L., Tardieu, J., Gaillard, P., Woisard, V., \& Ruiz, R. (2015). Relationship between speech intelligibility and speech comprehension in babble noise. Journal of Speech, Language, and Hearing Research, 58, 977-986.

Fox, C. M., \& Boliek, C. A. (2012). Intensive Voice Treatment (LSVT LOUD) for children with spastic cerebral palsy and dysarthria. Journal of Speech, Language, and Hearing Research, 55, 930-945.

Fox, C. M., Ebersbach, G., Ramig, L., O., \& Shapir, S. (2012). LSVT LOUD and LSVT BIG: Behavioral Treatment Programs for Speech and Body Movement in Parkinson Disease. Retrieved from http://dx.doi.org/10.1155/2012/391946

Frass, M. R. (2003). Towards intelligibility testing in dysarthria: A study of motor speech deficits in native Spanish speakers with Parkinson's disease (Doctoral Dissertation). Retrieved from http://etd.ohiolink.edu/view.cgi?acc num=ucin1051723241

Gamboa, J., Jiménez- Jiménez, F. J., Nieto, A., Montojo, J., Ortí-Pareja, M., Molina, J. A., García-Albea, E., \& Cobeta, I. (1997). Acoustic voice analysis in patients with Parkinson's Disease treated with dopaminergic drugs. Journal of Voice, 3, 314-320.

Goetz, C. G., Tilley, B. C., Shaftman, S. R., Stebbins, G. T., Fahn, S., Martinez-Martin, P., Poewe, W., Sampaio, C., Stern, M. B., Dodel, R., Dubois, B., Holloway, R., Jankovic, J., Kulisevsky, J., Lang, A. E., Lees, A., Leurgans, S., LeWitt, P. A., Nyenhuis, D., Olanow, C. W., Rascol, O., Schrag, A., Teresi, J. A., van Hilten, J. J., \& LaPelle, N. (2008). Movement Disorder Society-sponsored revision of the Unified Parkinson's Disease Rating Scale (MDS-UPDRS): Scale presentation and clinimetric testing results. Movement Disorders, 23, 2129-2170.

Hawks, J. W., Fourakis, M. S., Skinner, M. W., Holden, T., \& Holden, L. K. (1997). Effects of formant bandwidth on the identification of synthetic vowels by cochlear implant recipients. Ear \& Hearing, 18, 479-487.

Hoehn, M., \& Yahr, M. (1967). Parkinsonism: onset, progression and mortality. Neurology 17 (5), 427-42.

Hsu, S. C. (2017). The Effects of Intensive Voice Treatment on Speech Intelligibility and Acoustics of Mandarin Speakers with Hypokinetic Dysarthria Due to Parkinson's Disease (Doctoral dissertation). Retrieved from ProQuest Dissertations and Theses database. (UMI No. 10276316.)

Hustad, K. C. (2006). Estimating the intelligibility of speakers with dysarthria. Folia Phoniatrica et Logopaedica, 58, 217-228.

Jacobson, B. H., Johnson, A., Grywalski, C., Silbergleit, A., Jacobson, G., Benninger, M. S., \& Newman, C. W. (1997). The Voice Handicap Index (VHI): Development and validation. American Journal of Speech-Language Pathology, 6(3), 66-70.

Jiménez- Jiménez, F. J., Gamboa, J., Nieto, A., Guerrero, J., Ortí-Pareja, M., Molina, J. A., García-Albea, E., \& Cobeta, I. (1997). Acoustic voice analysis in untreated patients with Parkinson's Disease. Parkinsonism and Related Disorders, 3, 111-116.

Kang, U. J., \& Auinger, P. (2012). Activity enhances dopaminergic long-duration response in Parkinson disease. Neurology, 78, 1146-1149.

Kapsner-Smith, M. R., Hunter, E. J., Kirkham, K., Cox, K., \& Titze, I. R. (2014). A randomized controlled trial of two semi-occluded vocal tract voice therapy protocols. Journal of Speech, Language, and Hearing Research, 58, 535-549.

Kleim, J. A., \& Jones, T. A. (2008). Principles of experience-dependent neural plasticity: 
implications for rehabilitation after brain damage. Journal of Speech, Language, and Hearing Research, 51, 225-239.

Kleim, J. A., Jones, T. A., \& Schallert, T. (2003). Motor enrichment and the induction of plasticity before or after brain injury. Neurochemical Research, 28, 1757-1769.

Lansford, K. L., Liss, J. M., Caviness, J. N., \& Utianski, R. L. (2011). A cognitive-perceptual approach to conceptualizing speech intelligibility deficits and remediation practice in hypokinetic dysarthria. Parkinson's Disease, 2011, 1-9.

Lemos de Azevedo, L., Soares de Souza, I., Marques de Oliveira, P., \& Cardoso, F. (2015). Effect of speech therapy and pharmacological treatment in prosody of parkinsonians. Archivos de Neuro-psiquiatria, 73, 30-35.

Levy, E. S., Chang, Y. M., Ancelle, J., \& McAuliffe, M. (2017). Acoustic and perceptual consequences of speech cues for children with dysarthria. Journal of Speech, Language, and Hearing Research, 60, 1766-1779.

Levy, E. S., Moya-Gale, G., Hopf, R., Forrest, K., \& Ramig. L. O., (2016). Effects of soundpressure-level changes on sentence-intelligibility in adults with Parkinson Disease following two treatment approaches. American Speech Language and Hearing Annual Convention, Philadelphia, PA.

Liss, J. M., Utianski, R., \& Landsford, K. (2013). Crosslinguistic application of English-centric rhythm descriptors in motor speech disorders. Folia Phoniatrica et Logopaedica, 65, 319.

Lobo, A. Saz, P., Marcos, G., Día, J. L., de la Cámara, C., Ventura, T., Morales Asín, F., Fernando Pascual, L., Montañés, J. A., \& Aznar, S. (1999). Revalidación y normalización del Mini-Examen Cognoscitivo (primera version en castellano del Mini-Mental Status Examination) en la población general geriátrica. Medicina Clínica (Barc), 112, 767-774.

Martel Sauvageau, V., Roy, J.- P., Langlois, M. \& Macoir, J. (2015). Impact of the LSVT on vowel articulation and coarticulation in Parkinson's disease. Clinical Linguistics \& Phonetics, 2015, 1-17.

McAuliffe, M. J., Baylor, C. R., \& Yorkston, K. M. (2016). Variables associated with communicative participation in Parkinson's disease and its relationship to measures of health-related quality-of-life. International Journal of Speech-Language Pathology, 27, 1-11.

McAuliffe, M. J., Kerr, A. E., Gibson, E. M. R., Anderson, T., \& LaShell, P. J. (2014). Cognitive-perceptual examination of remediation approaches to hypokinetic dysarthria. Journal of Speech, language, and Hearing Research, 57, 1268-1283.

Magill, R. A. (2011). Motor learning and control: Concepts and applications $\left(9^{\text {th }}\right.$ ed). New York, NY: McGraw-Hill.

Miller, N., \& Lowit, A. (Eds.). (2014). Motor Speech Disorders: A Cross-Language Perspective. Tonawanda, NY: Multilingual Matters.

Moncher, F. J., \& Prinz, F, J. (1991). Treatment fidelity in outcome studies. Clinical Psychology Review, 11, 247-266.

National Parkinson Foundation (2013). Parkinson's Disease Overview. http://www.parkinson.org/parkinson-s-disease.aspx. Accessed June 16, 2013.

Neel, A. T. (2009). Effects of loud and amplified speech on sentence and word intelligibility in Parkinson Disease. Journal of Speech, Language, and Hearing Research, 52, 1021-1033.

Nolano, M., Provitera, V., Estraneo, A., Selim, M. M., Caporaso, G., Stancanelli, A., Saltalamacchia, A. M., Lanzillo, B., \& Santoro, L. (2008). Sensory deficit in Parkinson's disease: evidence of a cutaneous denervation. Brain, 131, 1903-1911. 
Núñez-Batalla, F., Corte-Santos, P., Señaris-González, B., Llorente-Pendás, J. L., Górriz-Gil, C., \& Suárez-Nieto, C. (2007). Adaptación y validación del índice de incapacidad vocal (VHI-30) y su version abreviada (VHI-10) al español. Acta Otorrinolaringológica Española, 58, 386-92.

Ozimek, E., Warzybok, A., \& Kutzner, D. (2010). Polish sentence matrix test for speech intelligibility measurement in noise. International Journal of Audiology, 49, 444-454.

Pinto, S., Chan, A., Guimarães, I., Rothe-Neves, R., \& Sadat, J. (2017). A cross-linguistic perspective to the study of dysarthria in Parkinson's disease. Journal of Phonetics, 64, 156167.

Ramig, L. O., Countryman, S., Thompson, L. L., \& Horii, Y. (1995). Comparison of two forms of intensive speech treatment for Parkinson Disease. Journal of Speech and Hearing Research, 38, 1232-1251.

Ramig, L.A., Levy, E.S., Fox, C.M., Halpern, A., Spielman, J., Moya-Gale, G., Goudarzi, A. (2015). Impact of LSVT LOUD and LSVT ARTIC on speech intelligibility in Parkinson's disease. Movement Disorders, 30, Suppl 1, S112.

Ramig, L. O., Sapir, S., Countryman, S., Pawlas, A. A., O’Brien, C., Hoehn, M., \& Thompson, L. L. (2001). Intensive voice treatment (LSVT) for patients with Parkinson's disease: a 2 year follow up. Journal of Neurology, Neurosurgery \& Psychiatry, 71, 493-498.

Rosen, K. M., Kent, R. D., Delaney, A. L., \& Duffy, J. R. (2006). Parametric quantitative acoustic analysis of conversation produced by speakers with dysarthria and healthy speakers. Journal of Speech, Language, and Hearing Research, 49, 395-411.

Sapir, S., Spielman, J. L., Ramig, L. O., Story, B. H., \& Fox, C. (2007). Effects of intensive voice treatment (the Lee Silverman Voice Treatment [LSVT]) on vowel articulation in dysarthric individuals with idiopathic Parkinson Disease: Acoustic and perceptual findings. Journal of Speech, Language, and Hearing Research, 50, 899-912.

Simpson, S. A., \& Cooke, M. (2005). Consonant identification in N-talker babble is a nonmonotonic function of N. Journal of the Acoustical Society of America, 118, 2775-2778.

Spielman, J., Ramig, L. O., Mahler, L., Halpern, A., \& Gavin, W. J. (2007). Effects of an extended version of the Lee Silverman Voice Treatment on voice and speech in Parkinson's disease. American Journal of Speech-Language Pathology, 16, 95-107.

Stipancic, K. L., Tjaden, K., \& Wilding, G. (2016). Comparison of intelligibility measures for adults with Parkinson's Disease, Multiple Sclerosis and healthy controls. Journal of Speech, Language, and Hearing Research, 59, 230-238.

Sussman, J. E., \& Tjaden, K. (2012). Perceptual measures of speech from individuals with Parkinson's disease and multiple sclerosis: Intelligibility and beyond. Journal of Speech, Language, and Hearing Research, 55, 1208-1219.

Tjaden, K., Kain, A., \& Lam, J. (2014). Hybridizing conversational and clear speech to investigate the source of increased intelligibility in speakers with Parkinson's Disease. Journal of Speech, Language, and Hearing Research, 57, 1191-1205.

Tjaden, K. \& Wilding, G. (2004). Rate and loudness manipulations in dysarthria: Acoustic and perceptual findings. Journal of Speech, Language, and Hearing Research, 47, 766-783.

Turner, G. S., Tjaden, K., \& Weismer, G. (1995). The influence of speaking rate on vowel space and speech intelligibility for individuals with Amyotrophic Lateral Sclerosis. Journal of Speech and Hearing Research, 38, 1001-1013. 
U.S. Census Bureau (2010). New Census Bureau Report Analyzes Nation's Linguistic Diversity.http://www.census.gov/newsroom/releases/archives/american_community_survey_acs/cb10-cn58.html. Accessed July 1, 2013.

Van Den Eèden S. K., Tanner, C. M., Bernstein, A. L., Fross, R. D., Leimpeter, A., Bloch, D. A.,\& Nelson, L. M. (2003). Incidence of Parkinson's disease: variation by age, gender, and race/ethnicity. American Journal of Epidemiology, 157, 1015-1022.

Van Engen, K. J., \& Bradlow, A. R. (2007). Sentence recognition in native- and foreign-language multi-talker background noise. Journal of the Acoustical Society of America, 121, 519-526.

Weismer, G., Jeng, J. Y., Laures, J. S., Kent, R. D., \& Kent, J. F. (2001). Acoustic and intelligibility characteristics of sentence production in neurogenic speech disorders. Folia Phoniatrica et Logopaedica, 53, 1-18.

Whitehill, T. L., Kwan, L., Lee, F. P.-H., \& Chow, M. -N. (2011). Effect of LSVT on lexical tone in speakers with Parkinson's Disease. Parkinson's Disease, 2011, 1-9.

Wilson, R. H., Abrams, H. B., \& Pillion, A. L. (2003). A word- recognition task in multitalker babble using a descending presentation mode from $24 \mathrm{~dB}$ to $0 \mathrm{~dB}$ signal to babble. Journal of Rehabilitation Research and Development, 40, 321-328.

Wilson, R. H., Bell, T. S., \& Koslowski, J. A. (2003). Learning effects associated with repeated word-recognition measures using sentence materials. Journal of Rehabilitation Research and Development, 40, 329-336.

Yorkston, K. M., Beukelman, D. R., Hakel, M., \& Dorsey, M. (2007). Speech Intelligibility Test for Windows. Lincoln, NE: Madonna Rehabilitation Hospital.

Yorkston, K. M., Beukelman, D. R., Strand, E. A., \& Hakel, M. (2010). Management of Motor Speech Disorders in Children and Adults. Austin, TX: PRO-EYorkston, K. M. (1996). Treatment efficacy: Dysarthria. Journal of Speech and Hearing Research, 39, S46-S57. 